The reason why circulating antibodies disappear remains to be established; one possibility is that the nodes draining the tumour become incapable of responding to the antigen(s), allowing spread to occur.

\section{Lymphocytes and Melanoma Cells}

More recently, Dr. Graham Currie, working in our unit at Sutton, has been studying the effect of lymphocytes on melanoma cells using the microtechnique of Takasugi and Klein. ${ }^{19}$ The results have been difficult to interpret because some normal lymphocytes are cytotoxic to melanoma cells in vitro. For this reason he has been concentrating on comparing the cytotoxic effect of lymphocytes before and after the patients have been given an irradiated autograft of melanoma cells. In several patients lymphocytes taken before immunization were not cytotoxic to their own melanoma cells; but six days after the patients had been given an autograft of irradiated tumour cells the lymphocytes showed considerable cytotoxicity. Interestingly, the cytotoxic action of these autologous lymphocytes is not abolished by treating the melanoma cells with autologous serum at the same time-in other words, we have failed to show a blocking effect of antibody in this system.

There has been no effect on the course of the disease in any of the patients we have immunized with irradiated cells.

\section{Acute Leukaemia}

As with malignant melanoma some evidence suggests that there may be host resistance to acute leukaemia, even if it is only rarely effective. The finding by Burchenal ${ }^{20}$ that 103 people in the world have had proved acute leukaemia and are alive and free from disease between 5 and 17 years later is of great interest. Some of these patients were given intensive chemotherapy, and some form of host resistance must be postulated.

There have been reports that antibodies to leukaemic cells are present in the serum of patients with acute leukaemia. ${ }^{21}$ We have been more concerned with attempts to show that there are tumour-associated antigens in human acute leukaemia and to study changes in the patient's lymphocytes following the injection of irradiated autografts of leukaemic cells. Dr. Raymond Powles, working in our department at the Institute of Cancer Research, has collected large numbers of leukaemic blast cells from the peripheral blood using the I.B.M. Continuous Blood Cell Separater and then tested their ability to stimulate the lymphocytes from the same patient once a remission has been obtained. His preliminary results have shown, firstly, that leukaemic blast cells will stimulate autologous lymphocytes, thus confirming the work of others ${ }^{22-24}$ that tumour-associated antigens exist on the cell surface; and, secondly, that the response is greater after immunization with autologous irradiated blast cells, thus providing some rationale for the specific immunotherapy used by Mathe in human leukaemia. ${ }^{25}$ His results will shortly be published.

In summary, firstly, undoubtedly tumour-associated antigens exist in at least some tumours in man, just as they do in those of animals. Secondly, it is possible to show a host response in the form of antibodies or reactive lymphoid cells following immunization. However, as Professor Alexander has just stated, with the exception of Mathé's results ${ }^{25}$ in maintaining remissions in acute lymphoblastic leukaemia, it is premature to speak of immunotherapy in man at present. The most which can be expected is that by mounting a powerful immune response against the tumour antigens a few malignant cells will be killed. The place for immunotherapy is after the classical methods of reducing the number of tumour cells to the absolute minimum-in other words, surgery, radiotherapy, or intensive chemotherapy.

\section{REFERENCES}

Alexander, P., British Medical fournal, 1970, 4, 484.

Sumner, W. C., Cancer, 1953, 6, 1040

3 Sumner, W. C., and Foraker, A. G., Cancer, 1960, 13, 79.

- Smithers, D. W., Clinical Radiology, 1962, 13, 132

Smithers, D. W., Annals of the Royal College of Surgeons of England, 1967, 41, 160.

- Baker, H. W., American Surgeon, 1964, 30, 825.

' Everson, T. C., Annals of the New York Academy of Sciences, 1964, 114, 721.

8 Smith, J. L., and Stehlin, J. S., jun., Cancer, 1965, 18, 1399.

- Lewis, M. G., and Kiryabwire, J. W. M., Cancer, 1968, 21, 876.

10 Lewis, M. G., and Johnson, K., British fournal of Dermatology, 1968, 80, 362 .

1 Bodenham, D. C., Annals of the Royal College of Surgeons of England, $1968,43,218$

12 Lewis, M. G., Lancet, 1967, 2, 921.

12 Lewis, M. G., Lancet, 1967, 2, 921 . Holmes, E. C., and Ketcham, A. S., Surgery, 1968, 64, 233 .
R. A., Holmes, E. C., and Ketcham, A. S., Surgery, 1968, 64, 233.

1 Oettgen, H. F., et al., fournal of the National Cancer Institute, 1968, $41,827$.

15 Muna, N. M., Marcus, S., and Smart, C., Cancer, 1969, 23, 88.

16 Lewis, M. G., et al., British Medical Yournal, 1969, 3, 547.

17 Ikonopisov, R. L., et al., British Medical fournal, 1970, 2, 752

18 Alexander, P., and Hall, J. G., Advances in Cancer Research, 1970, 13, 1

19 Takasugi, M., and Klein, Eva., Transplantation, 1970, 9, 219.

20 Burchenal, J. H., Cancer Research, 1967, 27, 2616.

21 Doré, J. F., et al., Lancet, 1967, 2, 1396.

22 Bach, M. L., Bach, F. H., and Joo, P., Science, 1969, 166, 1520

23 Fridman, W. H., and Kourilsky, F. M., Nature, $1969,224,277$.

24 Viza, D. C., Bernard-Degani, Ora, Bernard, Cl., and Harris, R., Lancet, $1969,2,493$.

25 Mathé, G., British Medical fournal, 1970, 4, 487.
The experimental detection of tumour-specific antigens in the plasma membrane of malignant cells has raised the possibility that immunological procedures could be used in treatment. Antigenic substances specific to tumour cells have also been identified either inside the cell or associated with the extracellular matrix. Since some of these are released into the circulation they may become of great practical interest for diagnosis but they are less likely to be useful in relation to immunotherapy.

The identification of tumour-specific antigens associated

* Professor of Radiobiology, Chester Beatty Research Institute, Belmont, Sutton, Surrey. with the cell membrane presents many technical problems in man. ${ }^{1}$ However, much has been learned about them in tumours and leukaemias of experimental animals. When the malignancy has been induced by chemical or physical agents then the tumour antigens are unique for each tumour and there is no cross-reactivity between the antigens of tumours induced by the same carcinogenic substance. On the other hand, all the tumours induced in animals by a particular oncogenic virus have the same new antigens, though they will be different for different viruses. Even so, it has not been possible to demonstrate tumour antigens in several tumours in mice and rats, and possibly not all malignant cells have such new antigens. 


\section{Why are Antigenic Tumours "Successful"?}

In the early stages of human malignant disease the host's immune defences are normal and any subsequent impairment in immunity results from the advance of the disease. ${ }^{2}$ In animg $=$ immunosuppression facilitates the induction of tunifunt by viruses but has only a small-if any-effect on themicat carcinogenesis. The fact that lethal tumours can be produced by transplantation-often with a very few cells-in normal animals illustrates that generalized depression of the immune response is not an essential feature in the aetiology of malignant disease.

Inability of the host to recognize the tumour antigen as foreign-in other words, true specific tolerance-is rare in animal tumours and may not occur at all. ${ }^{3}$ Usually the animal's immune system responds to the tumour antigens though not sufficiently to eradicate the disease. ${ }^{4}$

It has been suggested that the tumour cells may "escape" being eliminated by the host defences because the host produces an antibody to the tumour-specific antigen present in the membrane. ${ }^{5}$ This is not cytotoxic (for example, does not bind complement) but coats the surface of the malignant cell and thereby prevents its destruction by other immune effector mechanisms. Nevertheless, there is no evidence that such a reaction actually occurs in vivo, and it seems unlikely that such "blocking" is a general phenomenon.

This is not to question the well-established phenomenon of immunological enhancement, which works in quite a different way. Here antiserum administered before transplantation facilitates the growth of an allograft in an immunologically foreign host by preventing effective immunization; the key fact is that in enhancement the antiserum must be given before the tumour. The reason why the existence of special phenomena such as immunosuppression, tolerance, or blocking antibodies are invoked arises from the misconception that in a normally functioning immune system all antigenic cells would be expelled. That the immune system is not so perfect is evident when one considers infectious diseases; tuberculosis, the growth of bacilli on the heart valve, or the disease resulting from the penicillin-resistant staphylococcus are not accounted for by special phenomena like tolerance or "blocking" antibodies, but occur because of imperfections inherent in immune defence.

\section{Effector Mechanisms}

Three effector mechanisms have been clearly recognized ${ }^{6}$ in the immunological reaction against tumours and in vivo all three probably co-operate; (1) humoral antibodies, which destroy tumour cells, probably in conjunction with complement; (2) the infiltration of the tumour by cytotoxic lymphoid cells, in a manner analogous to that which occurs during the rejection of homografts of normal tissue; and (3) macrophages, which play the main part in the rejection of mice ascitic leukaemias, ${ }^{7}$ killing the target cells by direct cell-to-cell contact. To be active in this way the macrophages must be coated with cytophilic antibody. Such cytotoxic macrophages can be recovered either from immunized animals or by exposing normal macrophages to spleen cells from immune animals. The latter release cytophilic antibody, which coats the macrophages and renders them cytotoxic.

The failure of the host to restrain an antigenic tumour arises because the effector mechanism of the immune response is confined by the limits imposed by different anatomical sites, as well as by the amounts of cytotoxic cells and antibodies that are potentially available.

Cytotoxic immune lymphoid cells have the capacity for traversing capillary beds and they can thus bring immune reactions to extravascular spaces. However, experimentally we have found that normally these cells are not discharged from a stimulated node into the circulation when the antigenic stimulus is a growing tumour. ${ }^{8}$ Circulating antibody is largely confined to the vascular compartment and probably is the principal effector mechanism for limiting blood-borne metastases. On the other hand, it is relatively powerless to control malignant cells at other sites. This would explain why patients with localized malignant melanoma had autoantibodies, whereas those with widely disseminated disease apparently had not. ${ }^{1}$

\section{Attempts at Immunotherapy}

The key conclusion from attempts at immunotherapy of tumours in animals is that a therapeutic effect is observed only if very few malignant cells are present at the time of treatment. ${ }^{9}$ Thus a useful effect can sometimes be observed only if there are fewer than 1,000 leukaemic cells in the mouse at the time of treatment.

\section{Use of Antiserum}

Specific antibodies directed against the tumour-specific membrane antigen and capable of killing leukaemic cells in the presence of complement are produced by immunizing foreign hosts with leukaemic cells. This is an unexpected finding because the host will respond to many other antigens present in the cell and one might have expected that it might not detect the weak tumour-specific antigens.

The use of antisera raised against the human patient's own leukaemic cells during remission (in other words, when there are few leukaemic cells) might be explored in acute leukaemia; immunosuppression by such sera due to antilymphocyte activity can probably be abolished by absorption against normal lymphocytes.

\section{Immune Lymphoid Cells}

Two approaches have been followed in using injections of immune lymphocytes for the treatment of tumours. In the first the tumour-bearing host is in a state of immunological suppression. As a result the injected lymphocytes proliferate unopposed in the foreign host and establish a graft-versus-host reaction. As part of this reaction the tumour cells are destroyed, but this action is not specific against the tumour. This procedure has found limited clinical applications but is hazardous because normal host cells are simultaneously destroyed.

The other approach is to inject immune lymphoid cells into an immunologically reactive host. The foreign lymphocytes do not grow, and there is no graft-versus-host disease so long as the recipient has normal immunocompetence. The cells responsible for the antitumour activity are the pyroninophilic blast cells-also called "immunoblasts"-which appear in the lymph draining a lypmh node between four and ten days after antigenic stimulation. They destroy the tumour cells by direct contact with them. There is no need for the injected cells to proliferate, or to survive for any length of time, in the tumour-bearing animal. Indeed, after irradiation with 1,000 r of $x$ rays immune lymphoid cells retain their antitumour activity but cannot proliferate; using such cells there is no danger of a graft-versus-host reaction even in an immunosuppressed animal. ${ }^{10}$

Recent experiments from several centres have raised the possibility that cytotoxic immunoblasts may be obtained by mixing circulating small lymphocytes with tumour cells in vitro. This clearly opens important new possibilities for the use of such cells in man.

\section{Autoimmunization}

Haddow and Alexander showed that autoimmunization with a piece of tumour obtained by biopsy and rendered incapable of growth by exposure to $x$ rays induced regression 
of primary sarcomas in rats if the amount of residual tumour was small." A similar phenomenon also occurs with some murine leukaemias, but again a detectable therapeutic effect can be observed only if there are few malignant cells. Possibly the local node draining a tumour becomes impaired by the massive quantity of antigen flowing into it and no longer discharges cytotoxic immunoblasts. ${ }^{8}$ This defect is local; by autoimmunization other nodes are stimulated and they release immunoblasts into the circulation, which are then able to attack the tumour. Whether this is the correct explanation or not, autoimmunization with irradiated tumour cells is effective in experimental animals in increasing their reaction to the tumour.

\section{Non-specific Stimulation of the Immune System}

The pioneering studies of Halpern and his associates showed that some unidentified components of Gram-negative organisms increase the resistance of animals against infection with bacteria and against increased resistance to a challenge with tumour cells. The best agents appear to be living B.C.G. or killed Corynebacterium paroum. Mathé showed in mice that these stimulants of the reticuloendothelial system are not only prophylactic (in other words, pretreatment with them increases resistance to a subsequent challenge) but that they can be used therapeutically in helping the host to eliminate a few tumour cells. ${ }^{12}$ We and others have confirmed these observations and a combination of treatment with B.C.G. and irradiated cells appears to act synergistically in some murine lymphomas.

We are testing the hypothesis that sometimes enough cytophilic antibody is formed but the capacity of the animal to reject tumour is limited by too few suitable macrophages. Non-specific stimulation by B.C.G. may increase the number of such macrophages. Tumours at or near the skin have been shown to disappear if inflammation is induced in the vicinity of the tumour. This was first observed by Gorer, who found that following infection such tumours sometimes disappeared ${ }^{6}$ In man cutaneous melanomas respond to vaccinia and basal cell carcinomas to painting with dichloronitrobenzene. All such procedures will bring macrophages into the vicinity of the tumour.

\section{Conclusions}

In carefully manipulated experiments various immunological procedures have a definite antitumour action, but it is quite premature to speak of clinical immunotherapy. With the exception of one study by Mathé there is no evicience that immunological procedures are useful in controlling malignant disease in man.

Since all the available cancer chemotherapeutic agents are immunosuppressive, it has been suggested that chemotherapy may be harmful. This I believe to be nonsense; so long as there is massive disease immunity can play no part. The first essential before immunological factors have the slightest chance of being effective is to reduce the amount of tumour present. If it were possible to achieve extensive cyto-reduction without immunosuppression obviously this would be the procedure of choice. However, even with the presently available agents it is much more important to kill tumour cells than to restrict chemotherapy in an attempt to preserve the immune system, especially since once the drugs have been withdrawn or reduced immune reactivity quickly returns.

\section{REFERENCES}

1 Fairley, G. H., British Medical Fournal, 1970, 4, 483.

2 Fairley, G. H., British Medical Fournal, 1969, 2, 467.

3 Allison, A. C., British Medical fournal, 1970, 4, 419.

4 Mikulsk. Z. B. Smith, C., and Alexander, P., Fournal of the National Cancer Institute, 1966, 36, 29.

Hellström, I., et al., Proceedings of the National Academy of Science, $1969,62,362$.

Gorer, P. A., Advances in Immunology, 1961, 1, 345.

7 Gorer, P. A., Advances in Immunology, 1961, 1, 345

7 Evans, R., and Alexander, P., Nature. In press. Alexander, P., Bensted, J., Delorme, E. J., Hall, J.
Proceedings of the Royal Society, 1969, B174, 237.

9 Alexander, P., Progress in Experimental Tumor Research, 1968, 10, 22

10 Alexander, P., and Hall, J. G., Advances in Cancer Research, 1970, 13, 1.

1 Haddow, A., and Alexander, P., Lancet, 1964, 1, 452

12 Mathe, G., British Medical fournal, 1970, 4, 487.

\title{
Immunotherapy of Cancer
}

\author{
Sir MICHAEL WOODRUFF, ${ }^{*}$ D.SC., F.R.C.S., F.R.S.
}

British Medical fournal, 1970, 4, 486-487

The questions we have to consider in this session may be stated as follows: "In so far as tumours possess associated antigens which are capable of evoking an immunological reaction in the patient or animal in which the tumour originated, can this be turned to therapeutic advantage, and if so, how?"

As a preliminary it is clearly important to consider how such tumours manage to survive at all. Fortunately Professor Klein has already dealt with this question very thoroughly, so I will content myself with one comment. The list of possible "escape mechanisms" which he proposed is not very different from lists put forward several years ago.". What has changed, thanks largely to the work of Klein himself, and some of his former associates like the Hellströms and the Möllers, is that we are today very much better equipped than we were to determine what particular escape mechanisms are operating in given animal models and even, to some extent, in individual patients.

* Professor of Surgery, University of Edinburgh.

\section{Possible Immunotherapeutic Procedures}

The list of possible immunotherapeutic procedures one might consider has also not changed very much over the past few years. In an Almroth W right lecture in $1964,{ }^{1}$ for example, I suggested that tumours which possessed specific antigens but had somehow escaped from control might be attacked by measures designed to intensify the host reaction, by passive immunization with antiserum, or by adoplive immunization with lymphoid cells from normal isogeneic donors or immunized donors of any strain or species. I went on to suggest that while, if a tumour lacked specific antigen, an attack could not be directed in the same way, it might perhaps be directed in a non-immunological way by concentrating the agent (antibody or cells) in the tumour, or by preliminary subsidiary procedures designed to make the tumour more susceptible than normal tissues to immunological attack. Alternatively, the immunological procedure might be nondirected (that is, might damage 